\title{
Different Scenarios Leading to Hyperchaos Development in Radiophysical Generators ${ }^{\dagger}$
}

\author{
Nataliya Stankevich \\ Higher School of Economics, National Research University, Nizhny Novgorod, Russia \\ + Presented at the Entropy 2021: The Scientific Tool of the 21st Century, 5-7 May 2021; Available online: \\ https://sciforum.net/conference/Entropy2021/.
}

Published: 30 April 2021

Chaos is a typical attribute of nonlinear dynamical systems in various fields of science and technology. One of the conventional indicator of chaotic dynamics is the largest Lyapunov exponent. Chaos is implemented in a situation when in the spectrum of Lyapunov exponents for a flow there is one positive, one zero and at least one negative exponents. Using full spectrum of Lyapunov exponents it is possible to classify hyperchaos, when spectrum contains two or more positive Lyapunov exponents.

In the frame of this work we describe two scenarios leading to occurrence of hyperchaos on the examples of the modified Anishchenko-Astakhov's generator and coupled generators of quasiperiodic oscillations. The first scenarios is a new scenario associated with appearance of Shilnikov's attractor, when saddle-focus with two-dimensional unstable manifold occurs via secondary Neimark-Sacker bifurcation and absorbs by chaotic attractor. For this scenario we will present cascade of secondary Neimark- Sacker bifurcations, corresponding to hierarchy of Shilnikov's attractors corresponding to hyperchaos. The second scenario was described previously, and associated with cascade of period doubling bifurcation of saddle-cycles with two-dimensional unstable manifold. Both scenarios will be presented for radiophysical generators.

The reported study was funded by RFBR according to the research project № 19-31-60030.

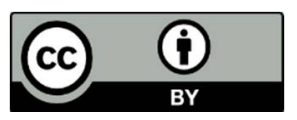

(C) 2021 by the author. Licensee MDPI, Basel, Switzerland. This article is an open access article distributed under the terms and conditions of the Creative Commons Attribution (CC BY) license (http://creativecommons.org/licenses/by/4.0/). 